\title{
Oscillations maintain grid position
}

Neural oscillations in the theta frequency range $(4-11 \mathrm{~Hz})$ have been suggested to be involved in the coding of an animal's spatial location, but how they do so has not been determined. Two papers published recently in Science now show that the proper functioning of a key cell type involved in spatial coding - the grid cell - requires theta oscillations.

Grid cells in the entorhinal cortex within the hippocampal formation exhibit intriguing firing properties that are thought to contribute to their role in coding spatial location. Grid cells fire when an animal is at specific locations in its surroundings. Their name derives from the regular hexagonal grid-like spacing of each cell's multiple firing locations across the animal's environment. In these experiments, the authors tested the theory that theta oscillations contribute to grid cells' distinctive firing patterns.

The authors reduced theta oscillations by disrupting the activity of the medial septum, an area that is central to the generation of theta rhythm in the hippocampal formation, using microinfusions of either lidocaine (Koenig et al.) or muscimol (Brandon et al.). They found that both the overall firing rate and the regularity of the spatial firing pattern (or 'gridness') of grid cells was reduced by the manipulations. By contrast, they did not observe changes in the functional properties of other cell types that contribute to spatial coding, including head-direction cells and place cells. Brandon et al. also examined the effect of reduced theta rhythms on conjunctive cells that have firing modulated by both grid position and head direction, and found a specific reduction in the cells' 'gridness' but no change in directional preference.

These studies show that rhythmic inputs from the medial septum to the hippocampal formation are required to maintain the grid-like pattern of firing in entorhinal grid cells,

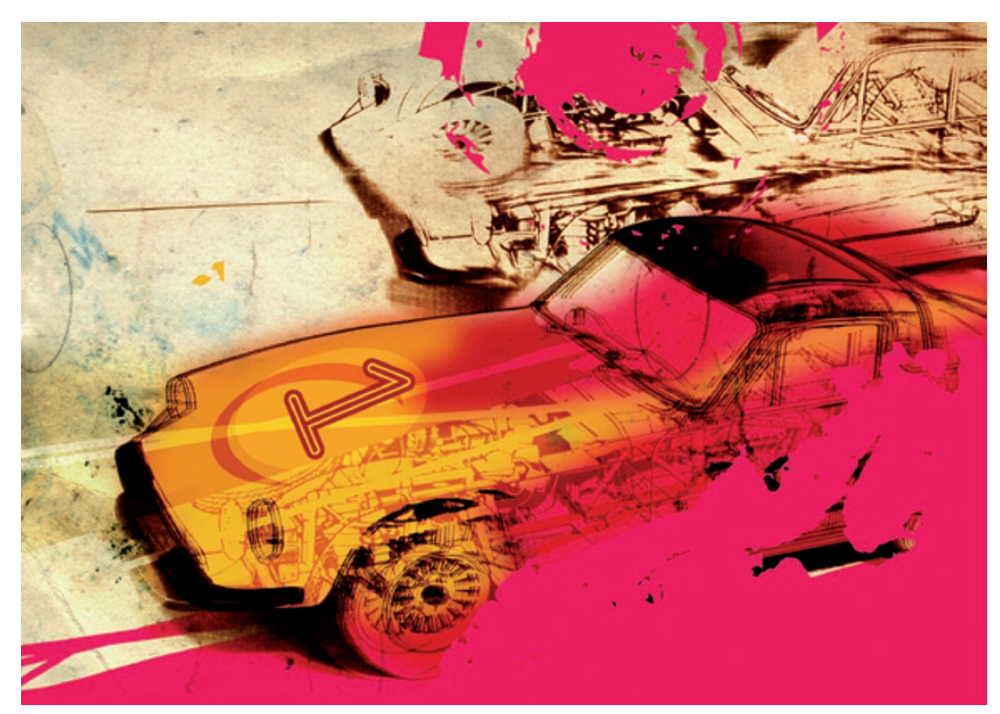

supporting the theoretical models that have suggested a role for theta oscillations in the generation of gridcell response patterns. These findings may have further importance for an understanding of the disrupted ability to locate oneself in space that occurs in some conditions, such as Alzheimer's disease.

Katherine Whalley

ORIGINAL RESEARCH PAPERS Koenig, J. et al. The spatial periodicity of grid cells is not sustained during reduced theta oscillations. Science 332, 592-595 (2011) | Brandon, M. P. et al. Reduction of theta rhythm dissociates grid cell spatial periodicity from directional tuning. Science 332, 595-599 (2011) 